Bolm Inst. oceanogr., S Paulo, 31(1):55-67, 1982

\title{
A INFLUÊNCIA DAS VARIAÇÕES NICTEMERAL E SAZONAL SOBRE AS CURVAS DE LUZ-FOTOSSİNTESE
}

\author{
Clövis TEIXEIRA
}

Instituto Oceanogräfico da Universidade de São Paulo

\section{Synopsis}

A series of experiments with natural phytoplankton populations was made to study the photosynthetic Pmax and $\alpha$ indices, under different phases of the diel cycles. By "in situ" and simulated experiments the exogenous and endogenous influences on the photosynthesis and total chlorophyll-a were studied. The results obtained indicate the diel variation can introduce a great deal of error in the determination of the primary production. The use of constant values for $\alpha$ and Pmax in the equation for the determination of primary production without carrying for the aspect above as well as for the seasonal variation may give results for the diumal values, with a very lon accuracy and without ecological significance. It was also observed that the measurements of chlorophyll-a varied within the relatively short period of time, required to perform a determination of photosynthesis. It is suggested that the Pmax, $\alpha$ and $I_{k}$ values used in primary productivity methodology should be revised, as the indices considered are based in chlorophyll concentration.

\section{Int rodução}

Um dos aspectos fundamentais indispensāveis para a obtenção de resultados significativos sobre a produção primäria de um ecossistema é, sem dúvida, a realização de estudos sobre a periodicidade nictemeral, não só das algas, como das características do meio ambiental. Segundo Ichimura (1968), os processos fisiológicos que ocorrem no fitoplâncton são influenciados pelos vários fatores ambientais, uma vez que estes mudam continuamente em termos de tempo e espaço.

A determinação da produção primária pode ser profundamente afetada pelo esquema experimental utilizado, não só em termos de duração do experimento em si, mas, principalmente, em relação ao período do dia em que o mesmo é executado.

0 principal objetivo deste trabalho é o de verificar o comportamento dos indices fotossintéticos Pmax e $\alpha$ em relação às diferentes fases do ciclo nictemeral e verificar algumas das influências exógenas e endógenas sobre a fotossintese e clorofila-a.

Além das variações cíclicas específicas, há o sério problema de como avaliar - significado da extrapolação, a partir de experimentos de curta duração para as produções diária, mensal e anual, através de modelos preditivos ou mesmo de

Publ. no 532 do Inst. oceanogr. da Usp. simples extrapolações. A existência de variações significativas da fotossíntese, em tempos curtos, comprova que a maioria dos métodos atualmente empregados para determinar a produção primäria diāria não deve apresentar suficiente precisão para expressar a produção primäria real.

\section{Material e mëtodos}

A área de estudo, denominada Enseada do Flamengo, está localizada na Lat. $23^{\circ} 30^{\prime}$ S Long. $45^{\circ} 06^{\prime} \mathrm{W}$, Brasil (Fig. 1). As coletas foram feitas durante períodos determinados de um mesmo dia-06:00, 12:00, 18:00, 00:00 horas-, baseando-se em resultados prévios obtidos por Teixeira (1980). As amostras, foram tomadas apenas da superfície.

Para os experimentos fotossintéticos, foram utilizados frascos de "pyrex" (2 transparentes $=\mathrm{T}$ e 1 preto $=\mathrm{D}$ ), sendo adicionado, em cada um, uma ampola de $\mathrm{NaH}^{14} \mathrm{CO}_{3}$ com atividade de $10 \mu \mathrm{Ci}$ (New England). No início de cada experimento, foi sempre inoculado um frasco com ${ }^{14} \mathrm{C}$ e filtrado imediatamente, sendo considerado "branco". A incubação foi feita durante um período de duas horas, seguida de imediata filtração, sob baixa pressão negativa ( $1 / 3 \mathrm{~atm}$.$) , sempre$ em série de três frascos, concomitantemente $(2 \mathrm{~T}+1 \mathrm{D})$, utilizando-se filtros 
"HA" da marca "Millipore" de $22 \mathrm{~mm}$ de diâmetro.

As amostras incubadas à superfície do mar ("in situ" - simulada) foram submetidas a um gradiente de energia solar, obtido através de uma série de filtros neutros (telas de náilon preto) colocados sobre os frascos num incubador, com gavetas pintadas totalmente de preto. Sob condições simuladas, a exposição das amostras à energia luminosa foi feita empregando-se um incubador tipo

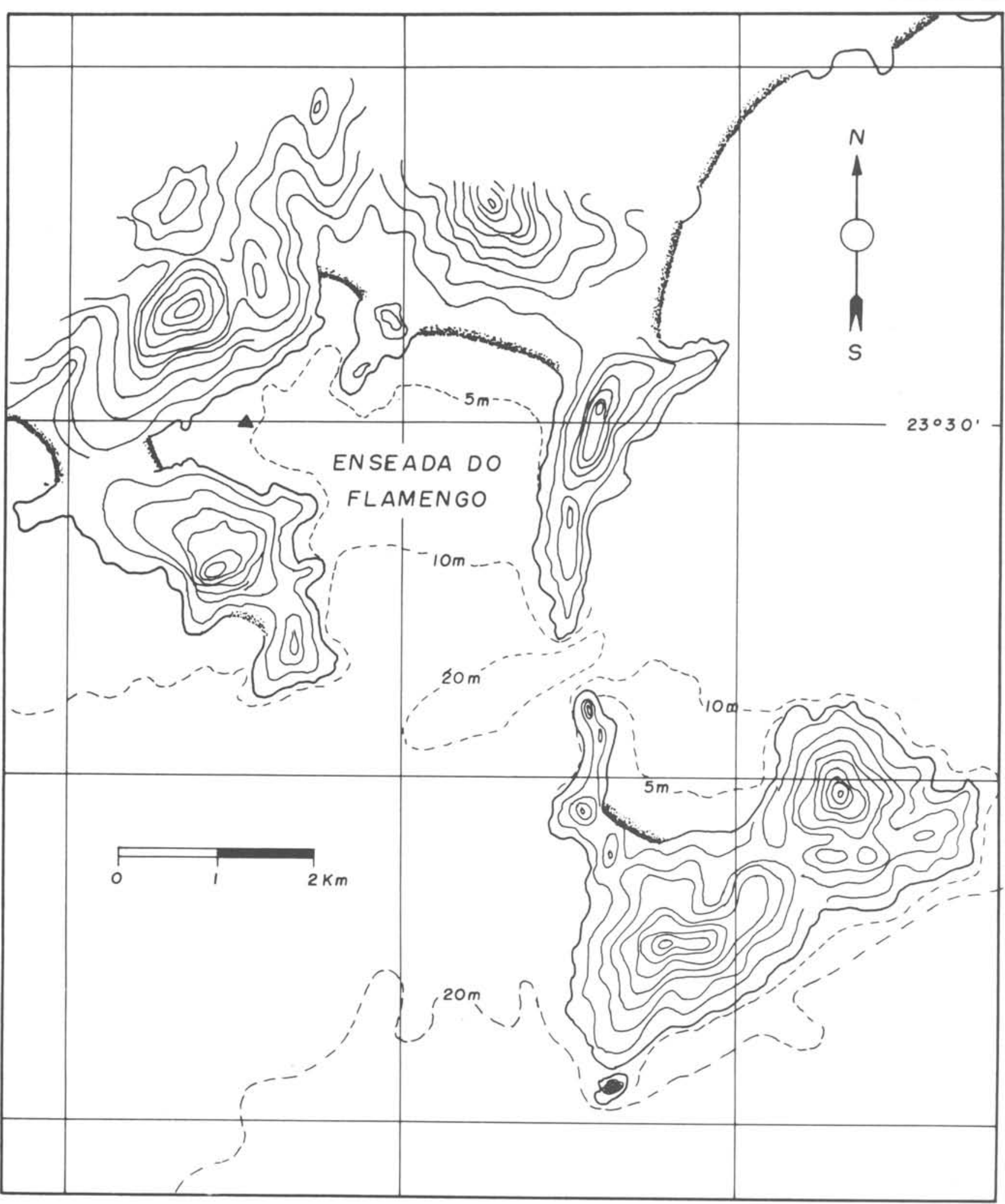

Fig. 1. Mapa da região, mostrando a posição da estação fixa. 
"Hawaii" com uma fonte de energia luminosa-constituída por seis lâmpadas Philips-W/33, de 20 Watts, para cada compartimento -, proporcionando um fluxo de energia luminosa de $30 \cdot 10^{15}$ quanta. $\mathrm{cm}^{-2} \cdot \mathrm{seg}^{-1}$, determinado por meio de um "quantum-meter" - mode1o Lambda - LI 185. Apenas para alguns experimentos, foi utilizado, também, outro incubador similar, porém com lâmpadas incandescentes (5 1âmpadas de 250 Watts-Philips) que proporcionaram um fluxo de energia luminosa da ordem de $55 \cdot 10^{15}$ quanta. $\mathrm{cm}^{-2}$. $\operatorname{seg}^{-1}$.

A radioatividade das amostras foi determinada num espectrômetro de cintilação líquida, modelo TRI-CARB C-2425 da Packard, empregando-se o líquido cintilador BRAY (Bray, 1960), seguindo-se as instruções de Aidar-Aragão et al. (1980). A produção primária determinada foi sempre considerada em termos de carbono particulado, is to é, com a exclusão dos produtos orgânicos excretados ou perdidos durante a filtração. A concentração do ${ }^{12} \mathrm{CO}_{2}$ total das amostras foi considerada como sendo $90 \mathrm{mg} \mathrm{CO} / 1$.

Foram feitas correções para o efeito da discriminação isotópica e para a respiração, ocorridos durante o tempo experimental. Os valores da produção primária foram calculados, empregando-se as equações de Vollenweider (1969), sendo apresentados em termos de mgC.mg clorofil $a-a^{-1} \cdot h^{-1}$.

A quantidade de clorofila-a foi determinada segundo a técnica de Parsons \& Strickland (1963), não descontando-se os valores de feofitina, consistindo, portanto, de valores de clorofila-a total e não apenas da clorofila-a funcional.

A radiação solar foi medida por meio de um actinógrafo bi-metálico da R.

Fuess, seg. Robitzch calibrado contra um actinógrafo padrão. Os fluxos de energia solar são expressos em termos de cal. $\mathrm{cm}^{-2} \cdot \min ^{-1}$ e a radiação total, durante um período ou diāria, foi obtida através da integração das curvas registradas pelo actinógrafo.

A radiação fotossinteticamente ativa foi estimada através de uma redução da radiação solar global em cerca de $\pm 48 \%$ (Talling, 1957).

A temperatura da āgua foi determinada durante todos os períodos experimentais, com um termômetro com a precisão de $0,1^{\circ} \mathrm{C}$.

0 oxigênio dissolvido doi determinado pelo método de Winkler, descrito por Magliocca (1966), sendo feita a estimativa da saturação do oxigênio dissolvido baseando-se nas tabelas apresentadas por Murray \& Riley (1969, In: Riley \& Chester, 1971).

A salinidade foi determinada com um salinômetro da marca Kahlsico-Induction Salinometer - 118 WA-200, modelo no 21705.

Dos nutrientes inorgânicos, foram determinados apenas o fosfato e o nitrato. 0 fosfato inorgâniço dissolvido foi determinado segundo a técnica de Murphy \& Riley (1962), modificada por Strickland \& Parsons (1968), e o nitrato dissolvido foi determinado pelo método de Mullin \& Riley (1955).

\section{Resultados}

\section{P-I de amostras mantidas em condicões naturais}

As curvas de luz-fotossíntese foram determinadas para verificar as suas principais características em diferentes horários de um mesmo dia, isto é, em diferentes períodos do ciclo nictemeral. Foram levados em consideração, principalmente, os valores da Pmax, $\alpha$ e $I_{k}$.

Os maiores valores da Pmax (mgC. mg clorofila-a $]^{-1} \cdot h^{-1}$ ) ocorreram, quase sempre, durante o fotoperíodo das 12:00 às 14:00 horas e, os menores, das 18:00 às 20:00 horas. Em termos nictemerais, a variação da Pmax foi de 1,10 entre os valores 2,48 e 1,38 num mesmo dia e de 5,92 entre os valores 6,94 e 1,02, considerando-se todos os resultados obtidos "in situ"-simulado. Em condições simuladas, a variação foi de 4,32 entre 5,86 e 1,54 num mesmo dia e de 7,16 entre os valores 8,66 a 1,50 considerandose todos os resultados obtidos em condições simuladas (Figs 2-3; Tab. I). Os valores de $\alpha$ (mgC. [clorofila-a $]^{-1} \cdot h^{-1}$. energia) apresentaram uma variação máxima de 0,883 a 2,329 num mesmo dia e de 0,124 a 2,329 considerando-se todos os valores obtidos "in situ"-simulado; em condições simuladas, a variação máxima de $\alpha$ foi de 0,200 a 1,255 considerandose todos os valores das diferentes épocas (Figs 2-3; Tab. I).

Para o fator $I_{k}(=P \max / \alpha)$, os resultados variaram bastante quando considedos "in situ" - da ordem de duas até seis vezes durante o fotoperíodo. Em condições simuladas (Figs 2-3; Tab. I), a variação foi menos acentuada. 
abela I - Resultado da variação nictemeral da capacidade fotossintética (Pmax), em termos de mgC (mgCclorof. $-a)^{-1}$; de $\alpha$. (mgC[mg clorof. $-a]^{-1} \cdot \mathrm{h}^{-1} \times 2,0 \cdot 10^{-1} \mathrm{cal} \cdot \mathrm{cm}^{-2} \cdot \mathrm{min}_{-1}^{-1}$ em condições "in situ'l-simuladas e mgC[mg clorof. $-\left.a_{-}^{-}\right|^{-1}$. $\mathrm{h}^{-1} \times 3.4 \cdot 10^{15}$ quanta. $\mathrm{cm}^{-2} \cdot \mathrm{seg}^{-1} \mathrm{em}$ condições simuladas; para os valores de $\mathrm{I}_{\mathrm{k}}$, em termos de cal. $\mathrm{cm}^{-2} \cdot \mathrm{min}^{-1} \mathrm{e} \times 10^{15}$ quanta. $\mathrm{cm}^{-2} \cdot \mathrm{seg}^{-1}$

\begin{tabular}{|c|c|c|c|c|c|c|c|}
\hline \multirow{2}{*}{ Data } & \multirow[b]{2}{*}{ Hora } & \multicolumn{3}{|c|}{ "IN SITU" - SIMULADO } & \multicolumn{3}{|c|}{ SIMULADO } \\
\hline & & $\alpha$ & Pmax & ${ }^{\prime} k$ & $\alpha$ & $P_{\max }$ & ' $k$ \\
\hline \multirow{4}{*}{$05 / 07 / 79$} & $06: 00$ & 0,609 & 1,02 & 0,03 & 0,255 & 1,50 & 21,26 \\
\hline & $12: 00$ & 0,612 & $>2,04$ & $>0,07$ & 0,468 & 3,60 & 27.96 \\
\hline & $18: 00$ & - & - & - & 0,306 & 1,80 & 20,67 \\
\hline & $00: 00$ & - & - & - & 0,350 & 2,92 & 19,22 \\
\hline \multirow{3}{*}{$24 / 10 / 79$} & $06: 00$ & 0,276 & 1,52 & 0,11 & & & \\
\hline & $12: 00$ & 0,124 & 2,48 & 0.27 & & & \\
\hline & $16: 00$ & 0,292 & 1,38 & 0,09 & & & \\
\hline \multirow{4}{*}{$26 / 02 / 80$} & $06: 00$ & 2,329 & 2,74 & 0,02 & 0,700 & 4,38 & 23,30 \\
\hline & $12: 00$ & 0,883 & $>3,68$ & $>0,11$ & 0,824 & 6,34 & 29,12 \\
\hline & $18: 00$ & - & - & - & 0,515 & 3,68 & 25.33 \\
\hline & $00: 00$ & - & - & - & 1,255 & 8,66 & 24,17 \\
\hline \multirow{4}{*}{$13 / 03 / 80$} & $06: 00$ & 1,800 & $>4,04$ & $>0,04$ & 0,761 & 5,86 & 27.27 \\
\hline & $12: 00$ & 0,550 & 6,94 & 0,25 & 0,528 & $>4,40$ & $>30,19$ \\
\hline & $18: 00$ & - & - & - & 0,351 & $>3,06$ & $>30,19$ \\
\hline & $00: 00$ & - & - & - & 0,200 & 1,54 & 26,49 \\
\hline
\end{tabular}

P-I de amostras mantidas em condições de taboratorio

Para as amostras que permaneceram sob iluminação constante, recebendo energia luminosa de $9,0.10^{15}$ quanta. $\mathrm{cm}^{-2} \cdot \mathrm{seg}^{-1}$, - valor da Pmax variou de 1,36 a 6,80; $\alpha$ variou de 0,217 a 0,470 e o $I_{k}$ variou de 28,15 a $48,10^{15}$ quanta. $\mathrm{cm}^{-2} \cdot \mathrm{seg}^{-1}$ (Fig. 4; Tab. II). A concentração da clorofila-a variou de 1,84 a 6,64 $\mathrm{mg} \cdot \mathrm{m}^{-3}$ (Fig. 5).

Radiacão solar fotossinteticamente ativa A radiação solar apresenta, tipicamente, valores máximos durante o verão e mínimos durante o inverno (Tab. III), ocorrendo, geralmente, uma acentuada assimetria no ciclo solar diário, fato este de grande importância ecológica. Durante o dia 24 de outubro, utilizando-se uma placa refletora, foi obtido um valor $50 \%$ acima do real e, portanto, alta intensidade de energia radiante (Tab. III).

\section{Oxigênio dissolvido (OD)}

Os resultados obtidos para o OD mostraram uma pequena variação nictemeral, com resultados 1 igeiramente maiores durante o fotoperíodo ( $\mathrm{Tab}$. IV). Tais resultados, parecem indicar a influência da produção primária do fitoplâncton sobre a quantidade do oxigênio dissolvido. Em termos de saturação, a variação foi de $75,55 \%$ a $80,86 \%$ durante o inverno e $85,37 \%$ a $96,66 \%$ durante o verão ( $\mathrm{T} a b$. IV).

\section{Salinidade}

Os valores da salinidade apresentaram uma pequena variação, de um mínimo de $32,81 \%$ o a um máximo de $34,35 \%$ 。

(Tab. IV), não apresentando nenhuma 

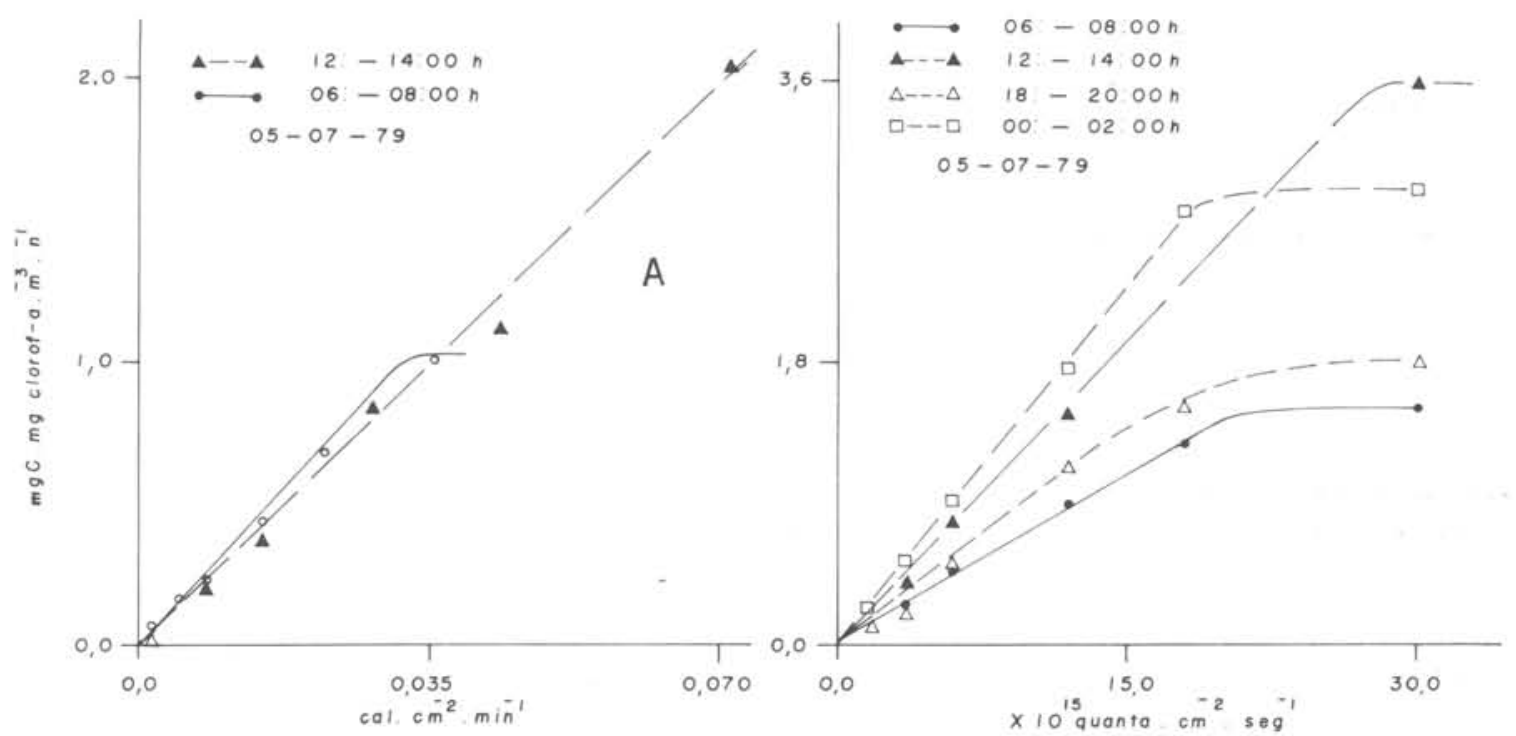

B

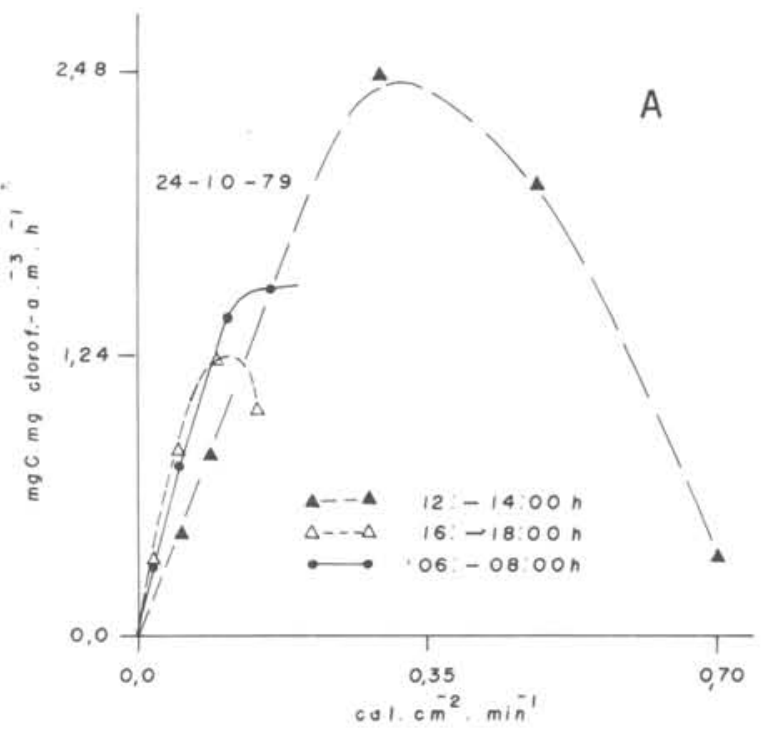

Fig. 2. Variação nictemeral da produção primäria $(p-I)$, em população natural de fitoplâncton. $A=$ "in situ"l-simulado; $B=$ simulado.

correlação com os meses de maior pluviosidade, uma vez que a região não recebe nenhum curso de àgua doce com vazão significativa.

\section{Temperatura da água}

A temperatura apresentou diferenças bem marcadas em termos sazonais, mas, em termos diärios, a variação foi pequena, alcançando um máximo sempre às 15:00 h e um mínimo geralmente às $06: 00 \mathrm{~h}$ (Tab. IV). A maior diferença diária foi da ordem de $2,15^{\circ} \mathrm{C}$.
Nitrato e fosfato

Os nutrientes determinados mostraram uma variação nictemeral durante todos os dias estudados. Para o nitrato, foi encontrada uma diferença entre os valores máximos e mínimos num mesmo dia, desde 0,04 até 1,00 $\mu \mathrm{g}-\mathrm{at} / 1$, enquanto que o fosfato apresentou uma variação nictemeral desde 0,03 até $0,18 \mu \mathrm{g}-\mathrm{at} / 1$ num mesmo dia (Tab. V). Em termos sazonais, ambos os nutrientes apresentaram uma tendência crescente do inverno para o verão, com maiores valores durante o mês de março (Tab. V). 
Tabela II - Resultados da Pmax, $\alpha$ e I $I_{k}$ de fitoplâncton natural mantido em condições constantes de temperatura $\left(25,0^{\circ} \mathrm{C}\right)$ e de energia luminosa $\left(9,0,10^{15}\right.$ quanta. $\left.\mathrm{cm}^{-2} \cdot \mathrm{seg}^{-1}\right)$ e em condições no escuro

\begin{tabular}{cccccccc}
\hline Data & Hora & \multicolumn{3}{c}{ ILUMINADO } & \multicolumn{4}{c}{ NO ESCURO } \\
& & $\alpha$ & Pmax & $I_{K}$ & $\alpha$ & $P_{\max }$ & $I_{K}$ \\
\hline & $06: 00$ & 0,380 & 3,80 & 34,95 & - & - & - \\
$17104 / 80$ & $12: 00$ & 0,470 & 6,80 & 48,54 & 0,374 & 4,94 & 46,26 \\
& $18: 00$ & 0,325 & 3,62 & 37,86 & 0,239 & 3,16 & 29,10 \\
& $00: 00$ & 0,217 & 1,36 & 28,15 & 0,265 & 3,50 & 27,61 \\
\hline
\end{tabular}
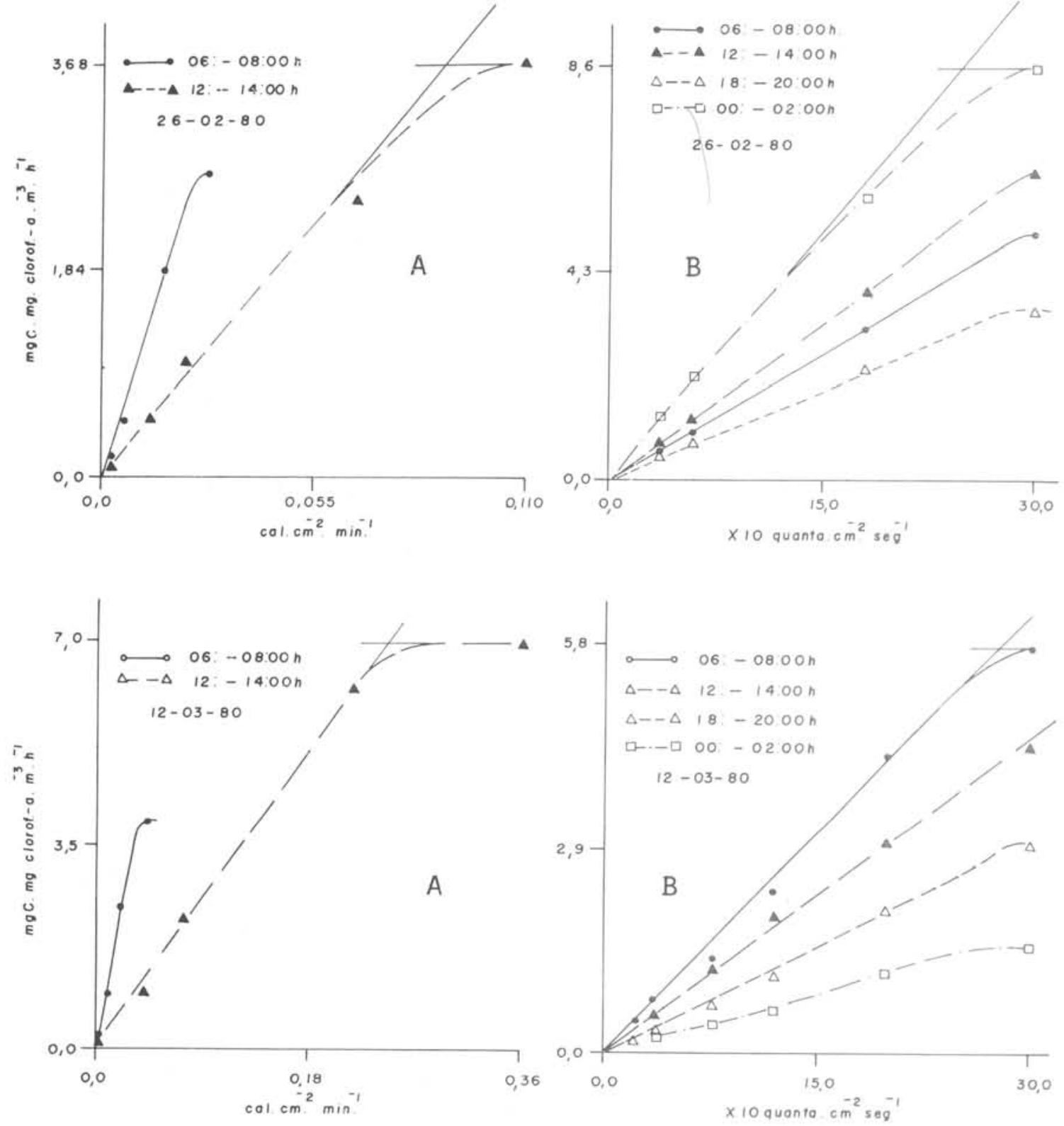

Fig. 3. Variação nictemeral da produção primária $(p-1)$, em população natural do fitoplâncton. $A=$ "in situ"l-simulado; $B=$ simulado. 


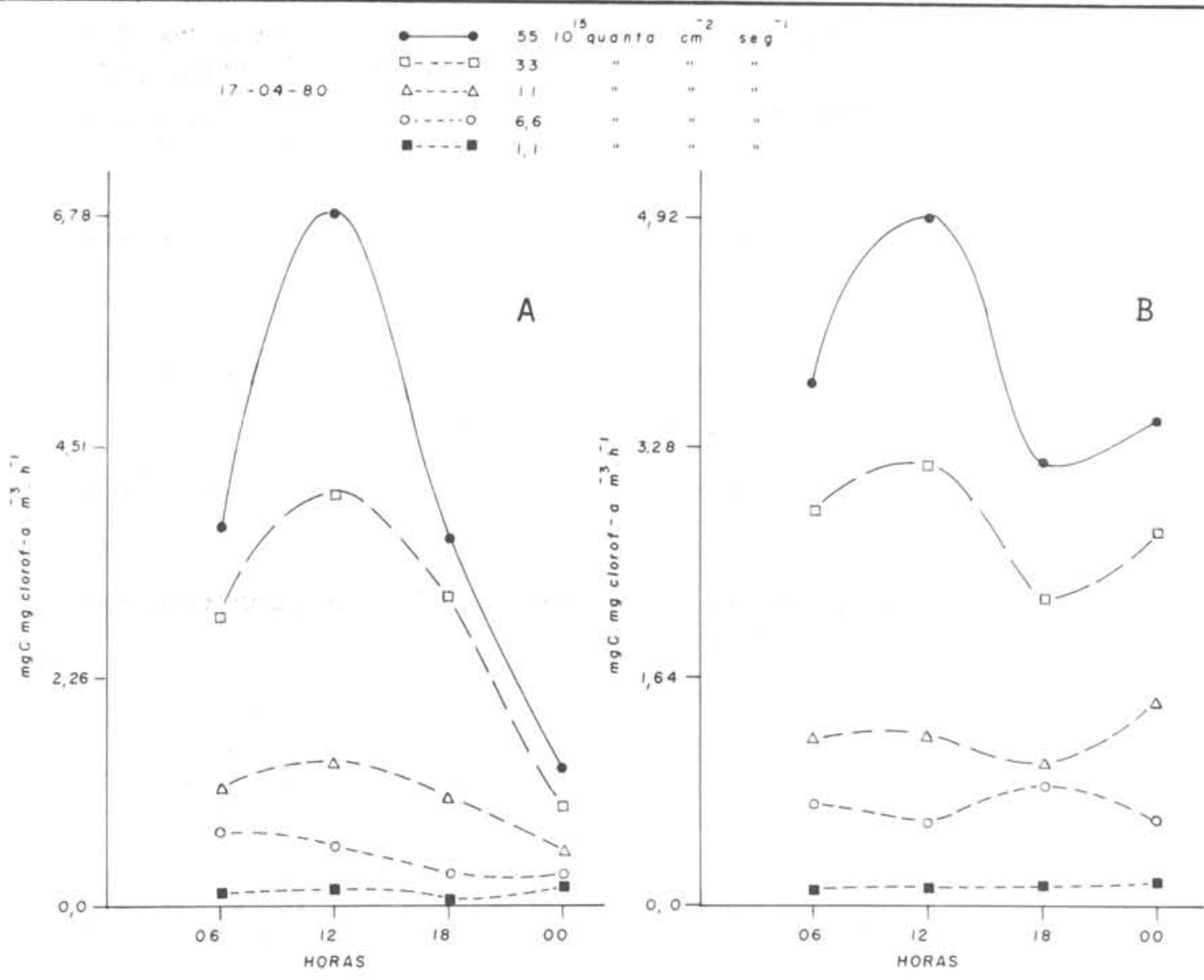

Fig. 4. Produção primäria mäxima (Pmax) em diferentes períodos do dia, em condições simuladas de energia luminosa, para duas populações mantidas em condições fóticas (A) e aföticas (B).

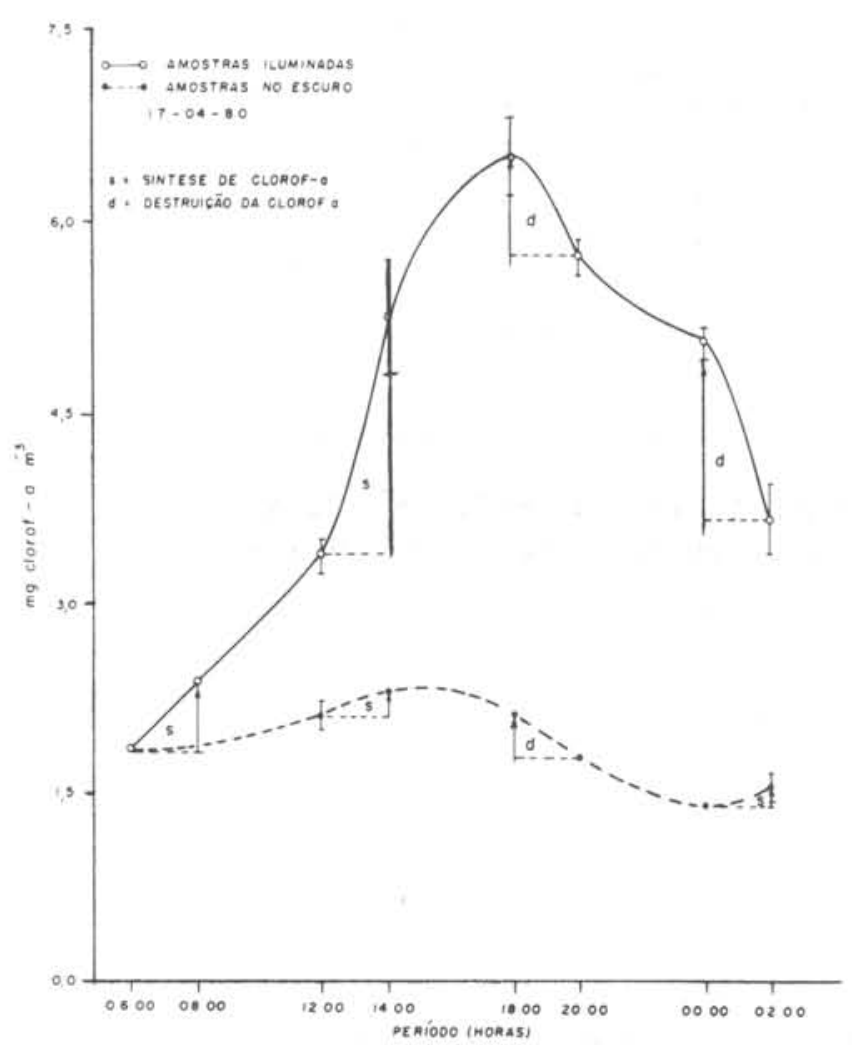

Fig. 5. Variação da clorofila-a em população natural de fi toplâncton, em condições experimentais constantes com relação à i luminação e à temperatura. 
Tabela III - Radiação solar fotossinteticamente ativa, em termos de $\mathrm{cal} . \mathrm{cm}^{-2}$, ocorrida durante diferentes períodos de um mesmo dia

\begin{tabular}{lccc}
\hline & $06: 00-08: 00$ & $12: 00-14: 00$ & $16: 00-18: 00$ \\
\hline $05 / 07 / 79$ & 0,038 & 0,070 & - \\
$24 / 10 / 79$ & 0,050 & 0,350 & 0,250 \\
$24 / 10 / 79$ & $0,057^{*}$ & $0,525^{*}$ & $0,375^{*}$ \\
$26 / 02 / 80$ & 0,142 & 0,185 & - \\
$12 / 03 / 80$ & 0,088 & 0,414 & - \\
\hline
\end{tabular}

(*) Resultados obtidos artificialmente com emprego de refletor

Tabela IV - Dados ambientais observados durante o período experimental

\begin{tabular}{|c|c|c|c|c|c|c|c|c|}
\hline \multicolumn{2}{|c|}{ Data } & \multicolumn{2}{|c|}{$05 / 07 / 1979$} & \multirow[b]{2}{*}{$T^{\circ} \mathrm{C}$} & \multirow[b]{2}{*}{$\mathrm{O}_{2}-\mathrm{m} 1 / 1$} & \multicolumn{2}{|c|}{$24 / 10 / 1979$} & \multirow[b]{2}{*}{$T^{\circ} \mathrm{C}$} \\
\hline Hora & $0_{2}-m 1 / 1$ & $\mathrm{O}_{2}-3$ & $5 \%$ & & & $\mathrm{O}_{2}-3$ & $5 \% 100$ & \\
\hline $06: 00$ & 4,00 & 77.52 & 13.76 & 21.95 & 3.99 & 80,12 & 32.81 & 24,10 \\
\hline $09: 00$ & 3,95 & 76.55 & 33,80 & 22,00 & 4,02 & 80,72 & 32.95 & 24,30 \\
\hline $12: 00$ & 4,03 & 78,10 & 33.78 & 22,30 & 4,07 & 81,72 & 33.06 & 24.50 \\
\hline $15: 00$ & 4,08 & 80,47 & 33,61 & 23,00 & 4,00 & 81,80 & 33.44 & 24.70 \\
\hline $18: 00$ & 4,10 & 80,86 & 33.67 & 23.00 & 3.98 & 79.91 & 33.95 & 24,00 \\
\hline $21: 00$ & 4,02 & $7 y .28$ & 33,83 & 23,00 & 3.83 & 76.90 & 33.74 & 23,90 \\
\hline $24: 00$ & 3.97 & 78,30 & 33.66 & 22,90 & 3.91 & 78,51 & 33.69 & 23.70 \\
\hline
\end{tabular}

\begin{tabular}{|c|c|c|c|c|c|c|c|c|}
\hline \multicolumn{2}{|c|}{ Data } & \multicolumn{2}{|c|}{$26 / 02 / 1980$} & \multirow[b]{2}{*}{$T^{\circ} \mathrm{C}$} & \multicolumn{4}{|c|}{$12 / 03 / 1980$} \\
\hline Hora & $0_{2}-m 1 / 1$ & $O_{2}-z$ & $5 \%$ & & $O_{2}-m 1 / 1$ & $0_{2}-2$ & $5 \%$ & $T^{\circ} \mathrm{C}$ \\
\hline $06: 00$ & 4,05 & 85,62 & 34,08 & 27.85 & 3,97 & 85.37 & 34.28 & 28,40 \\
\hline $09: 00$ & 4,03 & 86,66 & 34,12 & 28.30 & 4,11 & 91,33 & 34.35 & 29.35 \\
\hline $12: 00$ & 4,10 & 91.11 & 34.10 & 29,70 & 4.35 & 96,66 & 34.17 & 29,90 \\
\hline $15: 00$ & 4,19 & 93,11 & 34,15 & 30,00 & 4,28 & 95,11 & 34,09 & 30,15 \\
\hline $18: 00$ & 4,11 & 91.33 & 34.30 & 29,45 & 4.17 & 92,66 & 34,13 & 30,00 \\
\hline $21: 00$ & 4,04 & 88,40 & 34.25 & 29.15 & 4.10 & 91.11 & 34,22 & 30.00 \\
\hline $24: 00$ & 4,04 & 86,88 & 34,30 & 28,60 & 4,12 & 91,55 & 34,10 & 20,10 \\
\hline
\end{tabular}

Tabela V - Concentração de fosfato (ugat/1 : $\mathrm{P}-\mathrm{PO}_{4}$ ) e nitrato ( $\mu$ gat/1 : $\mathrm{N}-\mathrm{NO}_{3}$ ), obtidos durante o período experimental

\begin{tabular}{|c|c|c|c|c|c|c|c|c|}
\hline \multirow[b]{2}{*}{ Hora } & \multicolumn{2}{|c|}{$05 / 07 / 1979$} & \multicolumn{2}{|c|}{$24 / 10 / 1979$} & \multicolumn{2}{|c|}{$26 / 02 / 1980$} & \multicolumn{2}{|c|}{$12 / 03 / 1980$} \\
\hline & $P-P O_{2}$ & $\mathrm{~N}-\mathrm{NO}_{3}$ & $\mathrm{P}_{\mathrm{P}} \mathrm{PO}_{4}$ & $\mathrm{~N}-\mathrm{NO}_{3}$ & PAPO. & $\mathrm{N}-\mathrm{NO}_{2}$ & $P=P_{4}$ & $n-\mathrm{NO}^{\prime}$ \\
\hline $06: 00$ & 0,08 & 0,10 & 0,02 & 0,04 & 0,05 & 0.07 & 0.27 & 0,49 \\
\hline $09: 00$ & 0.07 & 0.09 & 0.03 & 0.03 & 0.04 & 0.08 & 0,19 & 0.23 \\
\hline $12: 00$ & 0.06 & 0.09 & 0,04 & 0.05 & 0.06 & 0.09 & 0,10 & 0,17 \\
\hline $15: 00$ & 0.09 & 0,14 & 0,02 & 0,04 & 0,07 & 0,09 & 0,14 & 0,23 \\
\hline $18: 00$ & 0,08 & 0.17 & 0.05 & 0.06 & 0.06 & 0.10 & 0.11 & 0,19 \\
\hline $21: 00$ & 0.07 & 0.16 & 0.04 & 0.05 & 0,07 & 0.14 & 0,09 & 1.17 \\
\hline $24: 00$ & 0,09 & 0,13 & 0,06 & 0,07 & 0,08 & 0,19 & 0,12 & 0,17 \\
\hline
\end{tabular}




\section{Discussão}

Uma curva de luz-fotossintese è uma expressão funcional resultante de todos os fatores ambientais que agem sobre a mesma, somados aos fatores endógenos específicos das diferentes espécies das comunidades fitoplanctônicas. Este tipo de curva é usada para determinar as características fisiológicas das espécies de algas ou de populações do fitoplâncton natural, por inúmeros autores (Rabinowitch, 1951; Talling, 1957; Yentsch \& Lee, 1966; Steemann-Nielsen \& J申rgensen, 1968; Teixeira, 1969; Tundisi, 1977; e outros). Baseando-se nas relações entre clorofila-a/fotossíntese e energia luminosa, adquirem-se dois parâmetros específicos de máxima importância: a fotossíntese em luz de saturação, $P \max \left(\mathrm{mgC}\right.$. $\left.\operatorname{mgCl}-a^{-1} \cdot \mathrm{h}^{-1}\right)$, e a eficiência fotossintética em baixa intensidade luminosa, $\alpha$ (mgC. [mgC1-a $]^{-1}$. $\mathrm{h}^{-1}$.energia). Estes dois parâmetros considerados constantes independentes das espécies e condições ambientais por autores como Dunstan (1973) e Bannister (1974), em relação a $\alpha$, e Ryther \& Yentsch (1958), em relação a Pmax -, foi demonstrado que variam significativamente (Platt \& Jassby, 1976).

Um fato de importância fundamental, entretanto, é o de a maioria dos autores não ter dado atenção aos efeitos nictemerais sobre as curvas de luz-fotossintese, particularmente aos de natureza endógena. Uma curva de luz-fotossíntese não é estática, nem para uma mesma população de fitoplâncton natural, nem para uma cultura unialgal quando relacionada com difentes períodos de um mesmo dia (MacCaul1 \& Platt, 1977; Gargas et al., 1979; Teixeira, 1980; Harding Jr. et al., 1981; Malone \& Neale, 1981).

Com relação ao indice $\alpha$ (Parsons et al., 1977), foram encontrados resu1tados entre 0,1 e $0,65 \mathrm{mgC}$. mg clorofi$1 \mathrm{a}-a \cdot \mathrm{h}^{-1}$ por KLUX e para Pmax valores entre 1,1 a $6,2 \mathrm{mgC}$. clorofila-a. $\mathrm{h}^{-1} \mathrm{em}$ culturas e entre 0,1 e 6,0 em amostras de fitoplâncton natural. Em termos nictemerais, Gargas et al. (1979) mostraram que o indice $\alpha$ variou de 1,0 a $1,6 \mathrm{mgC}(\mathrm{mgCl}-a)^{-1} \cdot \mathrm{h}^{-1} \cdot 2 \cdot 10^{-2} \mathrm{cal} \cdot \mathrm{cm}^{-2}$. $\min ^{-1}$. Encontraram, também, valores para Pmax, em termos nictemerais, da ordem de $6 \mathrm{mgC}(\mathrm{mgCl}-a)^{-1} \cdot \mathrm{h}^{-1}$, por volta do meio dia, e 2,5 mgC (mgC1-a) $)^{-1} \cdot \mathrm{h}^{-1}, \mathrm{du}-$ rante a noite. Mais recentemente, Harding et al. (1981) apresentaram resultados de pesquisas com culturas unialgais de diatomáceas, mostrando evidentes oscilações nictemerais, tanto para $\alpha$, como para Pmax. As oscilações mostraram ser dependentes da hora do dia, ocorrendo o valor máximo para $\alpha$ e Pmax no período da manhã ou perto do meio dia e um declínio, para baixos níveis, no período de escuro.

Malone \& Neale (1981) encontraram, para $P \max$, uma variação de 2,0 a 30,8 para o nanoplâncton e 0,3 a 24,0 para populações de "net"-plâncton; e para $\alpha$, de 0,011 a 0,153 para o nanoplâncton $e$ 0,002 a 0,154 para o "net"-p1âncton.

Os resultados deste trabalho apresentaram valores para $\mathrm{Pmax}$ (mgC. mgC1-a $]^{-1} \cdot \mathrm{h}^{-1}$ ) variando de 1,02 a 3,60 durante o inverno, e de 1,54 a 8,66 no verão (Tab. I), sendo que os valores relativos à amplitude máxima, em termos diurnos e em condições naturais, foram sempre obtidos entre as 12:00 e 14:00 h. Em condições simuladas, entretanto, embora o padrão de oscilação fosse similar, a amplitude mäxima ocorreu em diferentes horas, inclusive durante o período de escuro (Figs 2-3). Com relação aos valores de $\alpha(\mathrm{mgC} \text {. [mgCl-a }]^{-1} \cdot \mathrm{h}^{-1}$. $\left.2 \cdot 10^{-2} \mathrm{cal} \cdot \mathrm{cm}^{-2} \cdot \mathrm{min}^{-1}\right)$, foi encontrada uma variação de 0,550 a 2,329 , em condições naturais, e de 0,200 a 1,255 , em condições simuladas (Figs 2-3).

Segundo Gargas et al. (1979), apesar da confirmação da variação nictemeral dos parâmetros $P \max e$ e não são conhecidas, ainda, as causas do processo. Segundo Cur1 Jr. \& Sma11 (1965), as baixas razões de assimilação encontradas em comunidades naturais do fitoplâncton marinho foram causadas por deficiência de nutrientes. Thomas (1970) confirmou baixos valores para as razões de assimilação em águas deficientes em nitrogênio, enquanto que Glooschenko \& Cur1 Jr. (1971) comprovaram experimentalmente a influência dos nutrientes sobre as razões de assimilação.

Este trabalho apresentou valores para $P \max$ maiores durante o verão, da ordem de 2,74 a 6,94 "in situ" e de 1,54 a 8,66 em condições simuladas, enquanto que, nos demais meses (inverno e primavera), os valores foram de 1,02 a 2,48 "in situ" e de 1,50 a 3,60 , em condições simuladas. Observando-se os dados 
Bolm Inst. oceanogr., S Paulo, 31(1), 1982

de nutrientes das amostras estudadas, verificou-se que, realmente, as concentrações maiores de $\mathrm{P}^{-\mathrm{PO}_{4}}$ e $\mathrm{N}-\mathrm{NO}_{3}$ ocorreram durante o verão, principalmente em março, quando obteve-se, em condições naturais, valores de Pmax da ordem de 4,04 (06:00 h) e 6,94 (12:00 h). Estudos realizados nesta mesma ārea demonstraram que o nitrogênio é um dos principais fatores que influenciam a produção primäria (Teixeira, 1973; Teixeira \& Vieira, 1976; Teixeira \& Tundisi, 1981), parecendo, portanto, estar de acordo com os trabalhos de Thomas (1970) e

Glooschenko \& Cur1 Jr. (1971). Dentre os fatores exógenos em que constatou-se que houve influência sobre Pmax, além dos nutrientes, ficou evidenciada significativamente a ação da temperatura sobre Pmax, comprovando-se os resultados de Eppley (1972). Com relação à energia solar, ficou demonstrada a influência do comportamento assimétrico da curva diāria da radiação solar, além das diferenças quantitativas e qualitativas encontradas sazonalmente e que, obviamente, influem diretamente sobre a produção primäria (Teixeira, 1980). Os demais parâmetros hidrológicos estudados não apresentaram influência marcante sobre a produção primäria.

Além dos fatores ambientais levados em conta e acima discutidos (temperatura, nutrientes e radiação solar), deve ser considerada, também, a influência dos fatores endógenos, uma vez que os organismos mantidos em condições simuladas mostraram, em termos de unidade de clorofila-a total, sob a mesma intensidade e qualidade de energia luminosa, uma evidente flutuação nictemeral (Tab. II; Figs 4-5).

Populações naturais de fitoplâncton, quando submetidas a condições constantes de temperatura e energia luminosa em laboratório, mostraram uma flutuação nictemeral bem definida e semelhante àquela, altamente variáve1, obtida em condições naturais, particularmente em relação à radiação solar, o que parece sugerir uma interação de fatores endógenos. Estes resultados parecem concordar com vários autores que acham que a periodicidade fotossintética é controlada por um "relógio biológico", isto $\bar{e}$, endogenamente, enquanto que a magnitude, e talvez a sincronização das taxas fotossintéticas, é regulada pelas variāves ambientais (Sournia, 1974;
Prézelin \& Ley, 1980).

Experimentos realizados em condições simuladas, mantendo duas subamostras de uma mesma população sob condições fóticas e aföticas constantes, mostraram evidente flutuação da clorofila- $a$, independentemente da energia luminosa, quando considerado o padrão da oscilação. Nos organismos iluminados, a magnitude da flutuação foi muito maior, mas o padrão de flutuação foi praticamente o mesmo nos dois lotes de organismos, o que também sugere uma certa influếncia de fatores endógenos (Figs 45). Entretanto, não se pode apresentar uma indicação mais segura, uma vez que - tempo experimental aqui empregado não permitiu a verificação da continuidade da tendência da resposta do experimento. Além da flutuação da clorofila-a em condições constantes, ficou demonstrada, também, a ocorrência de uma flutuação da fotossintese em termos de unidade de clorofila-a e energia constante a partir de $33,10^{15}$ quanta. $\mathrm{cm}^{-2} \cdot \mathrm{seg}^{-1}$, tanto em amostras mantidas constantemente iluminadas $\left(9,10^{15}\right.$ quanta. $\left.\mathrm{cm}^{-2} \cdot \mathrm{seg}^{-1}\right)$, como no escuro. Tais resultados (Figs 4-5), parecem sugerir que, independentemente das condições exógenas (energia luminosa), os fatores endógenos controlam a periodicidade da clorofila- $a$ e da fotossintese. Parece que fatores endógenos são responsáveis pela periodicidade rítmica, proporcionando diferentes potencialidades às unidades fotossintetizadoras, mesmo em condições constantes de energia luminosa, sendo que a amplitude e a fase dos eventos estariam sujeitas à habilidade das células em reterem informações ambientais ou, pelo menos, que as populações estejam seguramente acopladas ao ciclo luz:escuro, Estes resultados concordam com as hipóteses, teorias e conclusões obtidas por Haxo \& Sweeney (1965), Has tings \& Sweeney (1958), Doty \& Oguri (1957), Sweeney (1960; 1969), McMurray \& Hastings (1972), Taguchi (1976), Prézelin \& Ley (1980) e outros.

Ainda recentemente, Yamada et al. (1979), trabalhando com Spatoglossum pacificum, verificaram que as enzimas que participam na fotoassimilação do $\mathrm{CO}_{2}$ apresentam uma variação diurna quanto às suas atividades, de acordo com o rítmo circadiano. Todas as atividades das enzimas mostraram um rítmo típico de aproximadamente 24 horas de 
periodicidade quando as algas eram cultivadas sob condições iluminadas ou no escuro, sendo apenas ligeiramente mudadas em condições artificialmente alteradas de luz e escuro. Os estudos de Yamada et al. (op. cit.), embora realizados com feofíceas macroscópicas, mostraram resultados comparáveis aos obtidos neste trabalho.

Baseando-se nos conhecimentos atuais, parece que uma tentativa para explicar as variações nictemerais da produção primária só tem significado quando se leva em conta, além dos fatores ambientais, o acoplamento com os fatores intrínsecos, a composição específica da população e a metodologia empregada. Em regiões tropicais como a nossa, a absoluta falta de conhecimentos sobre as características fisiológicas das espécies do fitoplâncton constitui um fator limitante a mais quanto à possibilidade de uma melhor compreensão da físioecologia fitoplanctônica.

Os resultados aqui apresentados, assim como os de Teixeira (1980), proporcionaram a indicação de que a determinação da produção primária diária, sem ser levada em conta a variação nictemeral, pode apresentar resultados com uma grande margem de erros. A utilização de valores constantes para $\alpha$ e para Pmax nas equações que determinam a produção primäria podem apresentar resultados sem nenhuma precisão, devendo-se levar em conta que os parâmetros $\alpha$ e Pmax variam não só sazonal, mas também nictemeralmente. Assim, conclui-se que os métodos presentemente usados para determinar a produção primária diária integral não proporcionam resultados precisos e ecologicamente significativos. Outro fato de grande importância, é com relação à variação da clorofila-a dentro de um tempo relativamente curto, entre o início e o fim do período experimental, o que leva a questionar: qual valor deve ser considerado unidade de pigmento em relação à fotossíntese: o inicial ou o final?

Esta variação da clorofila- $a$, observada por Gieskes et al., (1979) e Teixeira (1980), continua sendo investigada em comunidades algais naturais e em culturas, pois, se a mesma for confirmada como sendo universal, os parâmetros Pmax, $\alpha$ e $I_{k}$ terão de ser reavaliados.

\section{Agradecimentos}

A FINEP (Financiadora de Estudos e Projetos) e à O.E.A., pelo apoio financeiro à pesquisa. Ao CNPq (Conselho Nacional de Desenvolvimento Científico e Tecnológico), pela Bolsa de Pesquisador.

\section{Referências bibliogräficas}

AIDAR-ARAGÃO, E.; TEIXEIRA, C. \& VIEIRA, A. A. H. 1980. Produção primária e concentração de clorofila-a na costa brasileira (Lat. $22^{\circ} 31^{\prime} \mathrm{s}$; Long. $\left.47^{\circ} 57^{\prime} \mathrm{W}\right)$. Bolm Inst. oceanogr., S Paulo, 29(2) (no pre1o).

BANNISTER, T. T. 1974. Production equations in terms of chlorophy11 concentration, quantum yield, and upper limit to production. Limnol. Oceanogr., 19:1-12.

BRAY, G. A. 1960. A simple efficient liquid scintillator for counting aqueous solutions in liquid scintillation. Anal. Biochem., $1: 279-285$.

CURL Jr., H. \& SMALL, L. F. 1965. Variations in photosynthetic assimilation ratios in natural, marine phytoplankton communities. Limnol. Oceanogr., 10 (supp1.): 67-73.

DOTY, M. S. \& OGURI, M. 1957. Evidence for a photosynthetic daily periodicity. Limnol. Oceanogr., 2:37-40.

DUNSTAN, W. M. 1973. A comparison of the photosynthesis-light intensity relationship in phylogenetically different marine microalgae. J. exp. mar. Biol. Ecol., 13:181-187.

EPPLEY, R. W. 1972. Temperature and phytoplankton growth in the sea. Fishery Bull. Fish Wildl. Serv. U.S., 70:1063-1085.

GARGAS, E.; HARES, I.; MARTENS, P. \& EDLER, L. 1979. Diel changes in phytoplankton photosynthetic efficiency in brackish waters. Mar. Bio1., 52:113-122.

GIESKES, W. W. C.; KRAAY, G. W. \& BAARS, M. A. 1979. Current ${ }^{14} \mathrm{C}$ 
methods for measuring primary production: gross underestimates in oceanic waters. Neth. J. Sea Res., 13(1):58-78.

GLOOSCHENKO, W. A. \& CURL Jr., H. 1971. Influence of nutrient enrichment of photosynthesis and assimilation ratios in natural North Pacific phytoplankton communities. J. Fish. Res. Bd Can., 28:790-793.

HARDING Jr., L. W.; MEESON, B. W.; PREZZELIN, B. B. \& SWEENEY, B. M. 1981. Diel periodicity of phorosynthesis in marine phytoplankton. Mar. Bio1., 61:95-105.

HASTINGS, J. W. \& SWEENEY, B. M. 1958. A persistent diurnal rhythm of luminescence in Gonyaulax polyedra. Biol. Bul1. mar. biol. Lab., Woods Hole, 115:440-458.

HAXO, F. T. \& SWEENEY, B. M. 1955. Bioluminescence in Gonyaulax polyedra. In: Johnson, F.H., ed.The luminescence of biological sys tems. Washington, D. C., Am. Assoc. Adv. Sci., p. 415-420.

ICHIMURA, S. 1968. Phytoplankton photosynthesis. In: Jackson, D. F., ed. - Algal, man and the environment. Syracuse, :103-120.

MacCAUlL, W. A. \& PLATT, T. 1977. Die1 variations in the photosynthetic parameters of coastal marine phytoplankton. Limnol. Oceanogr., 22: 723-731.

MAGLIOCCA, A. 1966. Curso prätico de oceanografia: métodos e rotina para determinação de clorinidade, oxigênio, fosfatos, nitratos e $\mathrm{pH}$ da água do mar. São Paulo, Univ. S Paulo, Inst. Oceanográfico/UNESCO, 35p. (mimeogr.).

MALONE, T. C. \& NEALE, P. J. 1981. Parameters of light-dependent photosynthesis for phytoplankton size fractions in temperate estuarine and coastal environments. Mar. Bio1., 61:289-297.

MCMURRAY, L. \& HASTINGS, J. W. 1972. No desynchronization among four circadian rhythms in the unicellular alga, Gonyaulax polyedra. Science, 175: 1137-1138.

MULLIN, J. B. \& RILEY, J. P. 1955. The spectrophotometric determination of nitrate in natural waters, with particular reference to seawater. Ana1. Chim. Acta, 12:464-481.

MURPHY, J. \& RILEY, J. P. 1962. A modified single solution method for the determination of phosphate in natural waters. Anal. Chim. Acta, $27: 31-36$.

PARSONS, T. R. \& STRICKLAND, J. D. H. 1963. Discussion of spectrophotometric determination of marine plant pigments, with revised equations for ascertaining chlorophylls and carotenoids. J. mar. Res., 21:155-163.

; TAKAHASHI, M. \& HARGRAVE, B. 1977. Biological oceanographic processes. Oxford, Pergamon Press, 322p.

PLATT, T. \& JASSBY, A. D. 1976. The relationship between photosynthesis and light for natural assemblages of coastal marine phytoplankton. J . Phycol., 12:421-430.

PREZELIN, B. B. \& LEY, A. C. 1980. Photosynthesis and chlorophy11- $a$ fluorescence rhythms of marine phytoplankton. Mar. Biol., 55:295-307.

RABINOWITCH, E. I. 1951. Photosynthesis and related process. New York, N.Y., Interscience Pub1., vo1. 2, p. 6031208.

RILEY, J. P. \& CHESTER, R. 1971. Introduction to marine chemistry. London, Academic Press, 465p.

RYTHER, I. H. \& YENTSCH, C. S. 1958. The estimation of phytoplankton production in the ocean from chlorophy11 and light data. Limnol. Oceanogr., 2:281-286.

SOURNIA, A. 1974. Circadian periodicities in natural populations of marine phytoplankton. Adv. mar. Bio1., 12:325-389. 
STEEMANN-NIELSEN, E. \& J RRGENSEN, E. G. 1968. The adaptation of plankton algae. I. General part. Physiol. P1 antarum, $21: 401-413$.

STRICKLAND, J. D. H. \& PARSONS, T. R. 1968. A practical handbook of seawater analysis. Bul1. Fish. Res. Bd Can., (167):1-311.

SWEENEY, B. M. 1960. The photosynthetic rhythm in single cells of Gonyaulax polyedra. Cold Spring Harbor Symp. quant. Biol., 25:145-148.

\section{Transducing}

mechanisms between circadian clock and over rhythms in Gonyaulax. Can. J. Bot., 47:290-308.

TAGUCHI, S. 1976. Short-term variability of photosynthesis in natural marine phytoplankton populations. Mar. Biol., 37:197-207.

TALLING, J. F. 1957. Photosynthetic characteristics of some freshwater diatoms in relation to underwater radiation. New Phytology, 56(1): 29-50.

TEIXEIRA, C. 1969. Estudo sôbre algumas características do fitoplâncton da região de Cananéia e o seu potencial fotossintētico. Tese de Doutoramento. Universidade de São Paulo, Fac. Filos., Ciênc. Letras, 82p. (mimeogr.).

1973. Preliminary studies of primary production in the Ubatuba region. Bolm Inst. oceanogr., S Paulo, 22:49-58.

1980. Estudo quantitativo da produção primāria, clorofila-a e parâmetros abióticos em relação à variação temporal (Lat. $23^{\circ} 30^{\prime} \mathrm{s}$ Long. $\left.45^{\circ} 06^{\prime} \mathrm{W}\right)$. Tese de Livre-Docência. Universidade de São Paulo, Instituto Oceanogräfico, 243 p. (não publicada).
TEIXEIRA, C. \& TUNDISI, J. G. 1981. The effect of nitrogen and phosphorus enrichments on phytoplankton in the region of Ubatuba (Lat. $23^{\circ} 30^{\prime} \mathrm{S}$ Long. $\left.45^{\circ} 06^{\prime} \mathrm{W}\right)$, Brazil. Bolm Inst. oceanogr., S Paulo, 30(1):77-86.

- \& VIEIRA, A. A. H. 1976.

Nutrient experiment using

Phaeodactylum tricornutum as an assay organism. Bolm Inst. oceanogr., S Paulo, 25(1):29-42.

THOMAS, W. A. 1970. On nitrogen deficiency in tropical Pacific oceanic phytoplankton: photosynthetic parameters in poor and rich water. Limno1. Oceanogr., 15:380-385.

TUNDISI, J. G. 1977. Produção primāria, "standing-stock", fracionamento do fitoplâncton e fatores ecológicos em ecossistema lacustre artificial (Represa do Broa, São Carlos). Tese de Livre-Docência. Universidade de São Paulo (Ribeirão Preto), 409p. (mimeogr.).

VOLLENWEIDER, R. A. 1969. Primary production in aquatic environments. IBP Handbook N8 12, Blackwe11, Oxford, 213

YAMADA, T.; IKAWA, T. \& NISIZAWA, K. 1979. Circadian rhythm of the enzimes participating in the $\mathrm{CO}_{2}$ photoassimilation of a brown alga, Spatoglossum pacificum. Bot. mar., 22:203-209.

YENTSCH, C. S. \& LEE, R. W. 1966. A study of photosynthetic light reactions, and a new interpretation of sun and shade phytoplankton. J. mar. Res., 24:319-337.

(Manuscrito recebido em 29/set./1981; ace ito em $6 / a b r . / 1982$ ) 